\title{
DETERMINATION OF BEST LOW-FREQUENCY MICROWAVE ANTENNA APPROACH FOR FUTURE HIGH RESOLUTION MEASUREMENTS FROM SPACE
}

\author{
P. O'Neill, R. Bindlish, J. Piepmeier, D. Le Vine, D. Hudson, L. Li, G. Cruz-Ortiz, and D. Olney \\ NASA Goddard Space Flight Center, Greenbelt, MD 20771 USA \\ tel: 1-301-614-5773, fax: 1-301-614-5808, Peggy.E.ONeill@ nasa.gov
}

\begin{abstract}
Microwave remote sensing measurements at $\mathrm{L}$ band ( 1.2-1.6 GHz) of geophysical parameters such as soil moisture will need to be at higher spatial resolution than current systems (SMOS/ SMAP/ Aquarius) in order to meet the requirements of land surface, ocean, and numerical weather prediction models in the near future, which will operate at $\sim 9$ $15 \mathrm{~km}$ global grids and $1-3 \mathrm{~km}$ regional grids in the next few years. In order to make progress toward these needed spatial resolutions, advancements in technology are necessary which would lead to improved effective (i.e. equivalent) antenna size. An architecture trade study was conducted to quantitatively define the value and limits of different microwave technology paths, and to select the most appropriate path to achieve the high spatial resolution required by science in the future without sacrificing performance, accuracy, and global coverage.
\end{abstract}

Keywords (Index Terms) -- soil moisture, passive microwave, antenna technology, aperture synthesis, high spatial resolution, active/passive.

\section{INTRODUCTION}

Soil moisture is the key land surface hydrologic state variable that helps to control the Earth's water and energy balance, and it provides critical information to such applications as weather and climate predictions, agricultural forecasts, and hazards monitoring. Although NASA's SMAP and ESA's SMOS missions have pushed the spatial resolution at L-band for accurate soil moisture determination in all-weather conditions to $\sim 40 \mathrm{~km}$ [1], the next generation of numerical weather prediction and land surface models will operate at global scales of 9-15 km in the next few years (regional scales of 1-3 km), and will likely push global resolution scales to $1-5 \mathrm{~km}$ in the next decade (limited only by available computing power and the resolution of the needed input data streams) [2]. Additionally, desired temporal resolution requirements are approaching 1 day for many users. For NASA measurements from space to keep pace with the requirements of the global modeling community, advances in spatial resolution are needed while still maintaining radiometric performance and complete global coverage in a timely manner.

In addition to soil moisture, high-quality L-band radiometer measurements from space have been used to provide very useful information to other parameter estimations such as sea surface salinity, sea ice, and ocean winds $[3,4]$. The improvements in resolution targeted by this study for soil moisture investigations are mirrored by similar requirements for improved resolution in other disciplines (such as the need for better remote sensing of salinity in coastal zones and along ice edges). The results of this study are relevant across a wide range of Earth science application areas.

The expected outcome of the proposed study is a quantitative assessment of the limits to growth of three different microwave technologies while maintaining the needed science performance, and how such limits match up with the spatial resolution of science measurements needed by global models in the next decade.

\section{OBJECTIVE}

The main goal of this study was to determine the technology path which shows the most promise through a trade study analysis which determines the effective limits of three different antenna approaches: (1) what is the largest real aperture mesh reflector that is feasible to spin and control (mechanically and thermally)? [SMAP example in Fig. 1a]; (2) what is the largest aperture synthesis radiometer in either 1-D or 2-D that can meet the requirements for radiometric accuracy in a cost- 


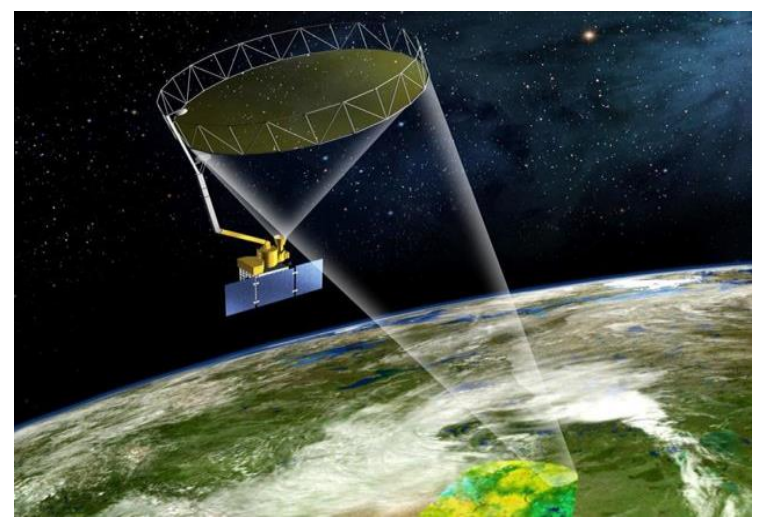

Fig. 1a. Large Spinning Mesh Reflector (SMAP example)

effective manner? [SMOS example in Fig. 1b]; and (3) where is the accuracy boundary between active-passive and passive-only approaches while reaching spatial resolution targets? While all three of these approaches have produced viable microwave measurements from space at relatively coarse resolution for accurate retrieval of soil moisture and other geophysical parameters, the limits to growth in the size of these antennas and hence the achievable spatial resolution in the future is currently unknown.

This study will examine the trade space between size and complexity versus performance, accuracy, swath coverage, etc. for each antenna approach. It will focus on defining the elements in common for large aperture designs (such as mechanical and thermal control of very large apertures) as well as those factors which are unique to the growth of a specific approach, such as location/design of the feed array for large mesh antennas, mutual coupling and impacts of thinning the array for aperture synthesis antennas, and the effects of radar noise on disaggregation algorithms for the active/passive approach. Each antenna technology will also be evaluated in terms of its ability to accommodate a radar if needed. Once a realistic limit to the size of a particular antenna type is determined, issues of cost, complexity, engineering control, and ability to meet the resolution and performance demands of the user communities will be factored in to achieve a recommendation for the most effective technology path to follow.

Although these efforts are focused on achieving the best path forward for a future high resolution L-band (1.2-1.4 GHz) mission (both ocean and land surface), the antenna technology trade results are also applicable to other low microwave frequencies and could help define the best approach for multi-frequency missions

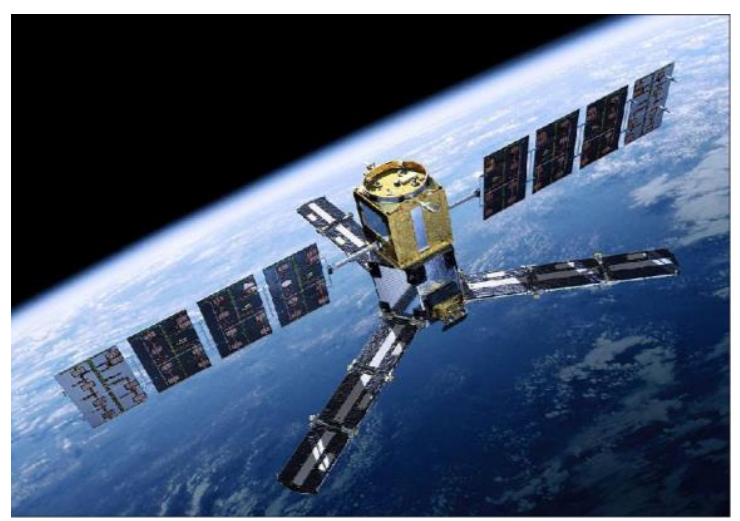

Fig. 1b. Large 2-D Aperture Synthesis Antenna (SMOS example)

(e.g., P- and L-band combination for accuracy and better penetration depth in the soil and vegetation, or L- and Ka-band combination to obtain coincident land surface temperature information needed in the soil moisture retrieval algorithm). However, for a multi-frequency mission, which is beyond the proposed scope of this study, the aperture design approach would have to be assessed to ensure that the specific microwave frequencies could be accommodated on the same antenna (for example, one early study found that an L$\mathrm{C}$ band combination would work on the basic HYDROSTAR 1-D aperture synthesis antenna, while an L-S band combination would not work [5]).

It is expected that the results of the proposed trade study will be relevant to the release of the next Earth Science Decadal Survey early in 2018. It is also relevant to an ongoing informal effort among European and U.S. scientists to coordinate L-band continuity activities.

\section{APPROACH}

Prior to studying the various microwave antenna technology approaches for a future high resolution mission, the basic science requirements for such a mission were laid out. These requirements included: (1) a revisit interval of 2-3 days, (2) complete global mapping within the revisit interval with no data gaps, (3) a spatial resolution of $10 \mathrm{~km}$ or better, including any incidence angle effects, and (4) a mission life of 2-3 years or longer. A future goal is a 1-day revisit with 1$5 \mathrm{~km}$ spatial resolution.

\subsection{Large Mesh Antennas}

The first technology approach is to utilize the SMAP spinning mesh reflector architecture (Fig. 1a) as a starting point and grow the antenna size, with or 
without a radar. The primary axis of the trade space is the reflector diameter. SMAP works very well with a 6-m diameter spinning mesh antenna. A larger antenna, however, would put additional demands on the spacecraft attitude control system (ACS) because of increased moment-of-inertia. One mitigation approach is to move the radiometer electronics and feed network off the spun side to the despun side of the spacecraft the resulting rotating polarization basis can be electronically compensated using existing techniques. One part of the study will be to determine if an ACS system could counter the dynamics of the larger spinning platform by requiring larger or more reaction wheels. At the same time, more balance mass might be required on the despun spacecraft such that the natural spinning motion of the entire system is more favorable for control purposes. Another part of the study will examine the trade between increased reflector size, spin rate, number of radiometer detectors, and instrument noise. An outcome of the analyses will be a high-level relationship between spacecraft resources, reflector size, and expected instrument performance.

\subsection{1-D or 2-D Aperture Synthesis Antennas}

The second technology approach involves the design of aperture synthesis antennas to provide large equivalent antenna sizes with less bulk, either in 1-D (HYDROSTAR example, Fig. 2a) or in 2-D (similar to SMOS, Fig. 2b). While these approaches permit efficient packing of the aperture in launch vehicle fairings for later deployment on orbit, there are a number of issues which limit their practical growth potential for high-quality science measurements. These include the thinning algorithm and number of baselines, the large number of small antenna elements/ correlations, the complexity of digital signal processing and image reconstruction, and noise/NEDT concerns. In particular, the major tradeoffs between spatial resolution and coverage will be explored since there is an inverse relationship between the aperture baselines (separation of antenna elements) and decorrelation at the Earth's surface which limits the field of view within which accurate science measurements can be obtained.

As part of the analysis of the aperture synthesis approach to large antennas, an effort will be made to quantify relationships between spatial resolution, positional knowledge of and number of antenna baselines, and accurate brightness temperature mapping. How minimizing NEDT for this antenna approach translates into engineering design considerations will also be examined, as well as lessons learned from SMOS [6]. The analyses should result in general formulas which will ingest a quantitative highlevel description of a microwave interferometer and output a rough estimate of its science performance.

\subsection{Adding a Radar to Microwave Radiometry}

The third technology approach is based on the assumption that a radiometer and radar can share the same antenna, and that there is a defined relationship between the radiometer and radar response at the same frequency for soil moisture or another geophysical parameter of interest that enables the process of disaggregating the radiometer brightness temperatures using simultaneous radar measurements to achieve improved spatial resolution (Fig. 2). Besides the added cost and complexity of flying a radar with a radiometer, radar observations have an inherent noise which impacts the disaggregation process and the accuracy of the resulting soil moisture estimates. As part of this study, assuming that a radar can be accommodated with a radiometer on the maximum size of each type of antenna studied, potential radar performance of each of the antenna types will be analyzed to determine if an active/passive approach is a simpler, less costly way of meeting the needed science performance and resolution requirements.

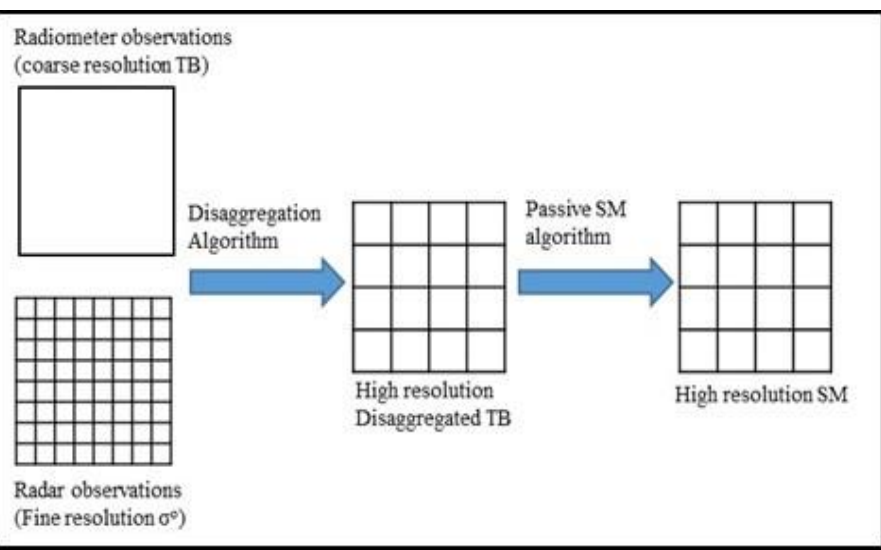

Fig. 2. Schematic diagram of the SMAP active/passive disaggregation algorithm methodology $[7,8]$.

\section{CONCLUSIONS}

Low frequency passive microwave missions require large apertures on orbit to achieve even moderate spatial resolution. Current concepts for a future high resolution soil moisture mission call for an overall effective antenna aperture of $\geq 20 \mathrm{~m}$, regardless of whether a real aperture or aperture synthesis approach is used, in order to achieve $10 \mathrm{~km}$ or better spatial resolution. Table 1 
Table 1. Comparison of Microwave Antenna Approaches for Future High Resolution Measurements from Space

\begin{tabular}{|c|c|c|}
\hline & Real Aperture & Synthetic Aperture \\
\hline Incidence Angle & Single angle conical scan & Multi-angle \\
\hline Noise & $\begin{array}{l}\text { Likely better than synthetic } \\
\text { aperture }\end{array}$ & Likely worse than real aperture \\
\hline Mechanical Considerations & $\begin{array}{l}\text { Needs mechanism for conical } \\
\text { scanning }\end{array}$ & No moving parts \\
\hline Bandwidth & $\begin{array}{l}\text { May be easier to go broadband } \\
\text { w/traditional feed structure }\end{array}$ & $\begin{array}{l}\text { May need more sophisticated } \\
\text { feed structure for broadband }\end{array}$ \\
\hline RFI & Know how to do RFI mitigation & $\begin{array}{l}\text { May need to develop RFI } \\
\text { mitigation technology }\end{array}$ \\
\hline Add $37 \mathrm{GHz}$ for $\mathrm{T}_{\mathrm{S}}$ estimation & Could use same antenna & Probably a separate instrument \\
\hline
\end{tabular}

presents a comparison of some antenna characteristics for real versus synthetic aperture approaches. Other considerations for future missions include the addition of another frequency (such as $37 \mathrm{GHz}$ for surface temperature estimation, a key parameter needed in soil moisture retrieval algorithms), the option of going broadband in frequency ( $\mathrm{L}$ to $\mathrm{P}$ band, for example), and the costs/benefits of adding an unfocused SAR to the primary radiometer instrumentation.

The expected outcome of this trade study when fully completed is an assessment of the limits to growth of three different microwave antenna technologies (real aperture, aperture synthesis, and active/passive approaches) and a recommendation to the science community on how best to proceed to enable future higher-resolution low frequency missions. However, the immediate path forward is likely to be dictated by the recommendations to NASA of the next Earth Science Decadal Survey (expected release in January 2018) as well as international efforts to coordinate Lband continuity or follow-on missions to SMAP, SMOS, and Aquarius.

\section{REFERENCES}

[1] Chan, S., R. Bindlish, P. O’Neill, E. Njoku, T. Jackson, A. Colliander, F. Chen, M. Burgin, S. Dunbar, J. Piepmeier, S. Yueh, D. Entekhabi, M. Cosh, T. Caldwell, J. Walker, X. Wu, A. Berg, T. Rowlandson, A. Pacheco, H. McNairn, M. Thibeault, J. Martínez-Fernández, Á. González-Zamora, M. Seyfried, D. Bosch, P. Starks, D. Goodrich, J. Prueger, M. Palecki, E. Small, J. Calvet, W. Crow, and Y. Kerr, "Assessment of the SMAP Level 2 Passive Soil Moisture Product," IEEE Trans. on Geoscience and Remote Sensing, vol. 54, no. 8, August 2016, pp. 4994-5007, doi: 10.1109/TGRS.2016.2561938.
[2] Personal communication: S. Belair/ Environment Canada, X. Zhan/ NOAA, R.Reichle/ GSFC GMAO, S. Kumar/ GSFC LIS, C. Peters-Lidard/ GSFC LIS, National Water Model; also ECMWF roadmap:

https://www.ecmwf.int/sites/default/files/ECMWF_Roadma p to $2025 . p d f$

[3] Le Vine, D. M., E. P. Dinnat, T. Meissner, S. H. Yueh, F.J. Wentz, S.E. Torrusio, (2015). "Status of AquariusSACD and Aquarius Salinity Retrievals", IEEE Journal of Selected Topics in Applied Earth Observations and Remote Sensing, 8(12): 5401-5415.

[4] Yueh, S., A. Fore, W. Tang, A. Hayashi, B. Styles, N. Reul, Y. Weng, and F. Zhang, "SMAP L-Band Passive Microwave Observations of Ocean Surface Wind During Severe Storms," IEEE Trans. on Geo. Rem. Sens., vol. 54, no. 12, Dec., 2016, pp. 7339-7350.

[5] HYDROSTAR design, Earth Science System Pathfinder proposal follow-on study, 1999.

[6] M. Martin-Neira et al., "Lessons Learnt from SMOS after 7 Years in Orbit," Proc. of IGARSS'17, IEEE, Fort Worth, Texas, July 23-28, 2017.

[7] Entekhabi, D., N. Das, E. Njoku, S. Yueh, J. Johnson, and JC Shi, SMAP Algorithm Theoretical Basis Document: Level 2 \& 3 Radar/Radiometer Soil Moisture (Active/Passive) Data Products. SMAP Project, D-66481, JPL, Pasadena, CA, Revision A, Dec. 9, 2014.

[8] Das, N., D. Entekhabi, and E. Njoku, "An Algorithm for Merging SMAP Radiometer and Radar Data for HighResolution Soil-Moisture Retrieval," IEEE Trans. on Geo. Rem. Sens., vol. 49, no. 5, May, 2011, pp. 1504-1512. 